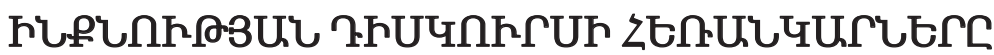 (Utipnnupuifulquif nhunuplnnưfitp)
}

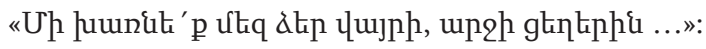 \\ પ.uhứ Stipjui

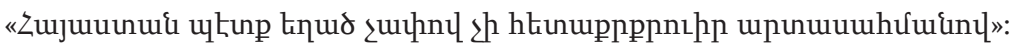 \\ uplynnnu Uupuqtiuk
}

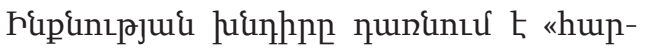

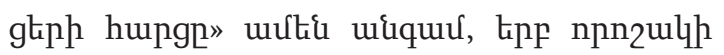
ulquifu hnumiphg htinn luaquinsu tiap

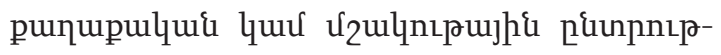
juil ungle, puiqug lupph phe pte zurn

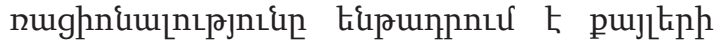

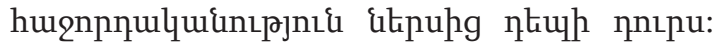

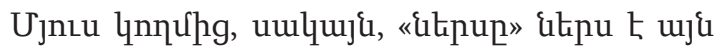

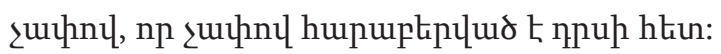
Ujl liknu' Elipup le npup uuhơuiuaqhon

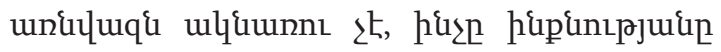

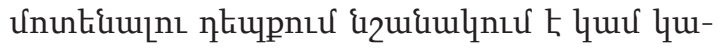

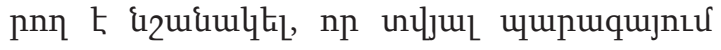

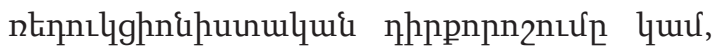

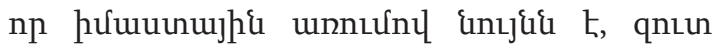

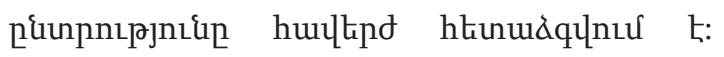

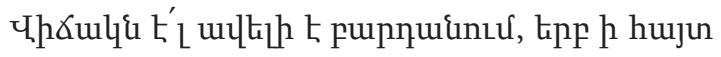
tis quihu punupulquid la punupulpparlyuis Uupunuhpultipiten, npnigg uqnkgnıpjui

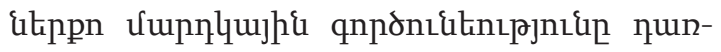

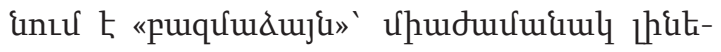
Łnl unupptip huppnıpjnı\&itinnıu: U.ju nh-

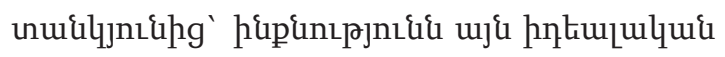

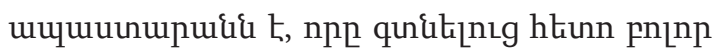

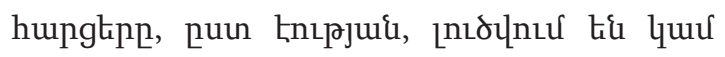

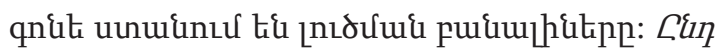

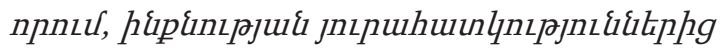

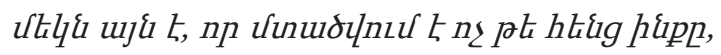

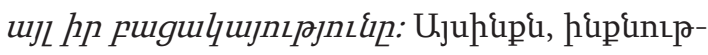

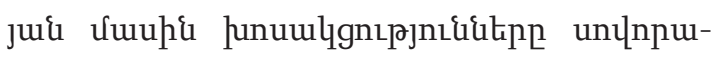

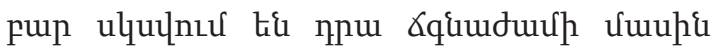

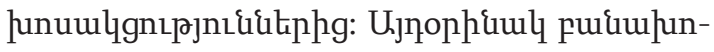

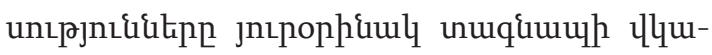

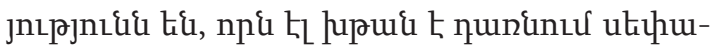

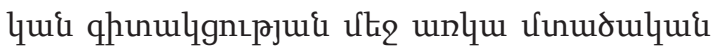

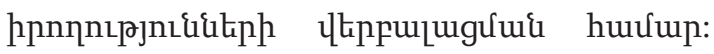

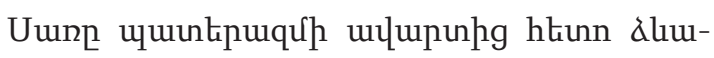

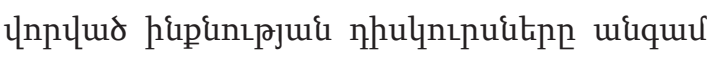

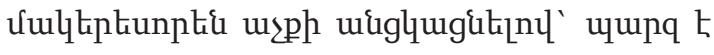

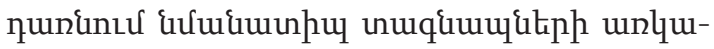

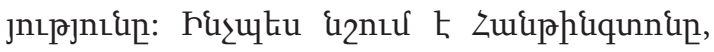

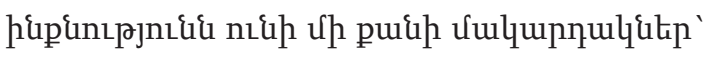

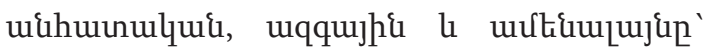
punupulnpulquif (nt'u, Huntington 1993:

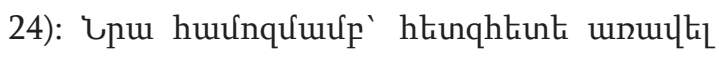

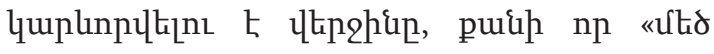

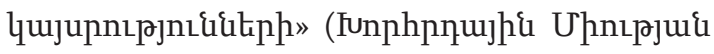

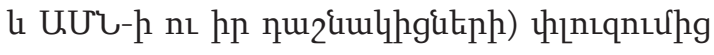
htiun tplpnitiph ungle dunugurl uju quu

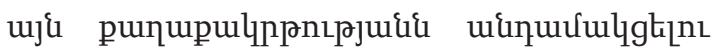

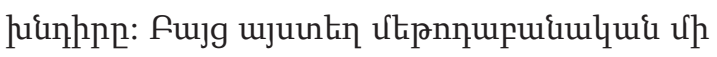

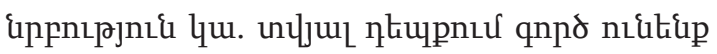

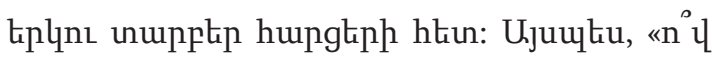
tư tu» hungn quinnn $t$ nplth unuppte

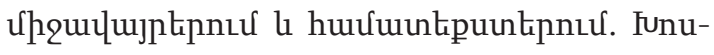

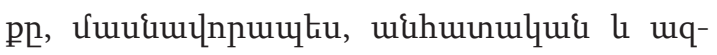




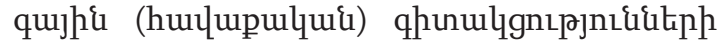

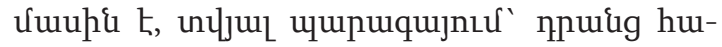

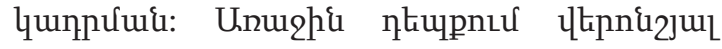
hupgn, np uflqupu upunuphi uqnulqutphg,

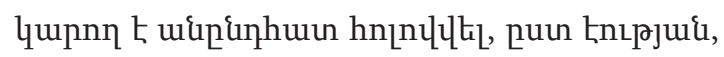

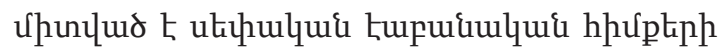

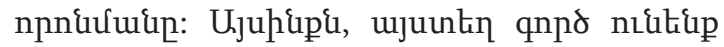
"hipqunıрjui»» «nıph2hg unuppknцtinı, hting hipp hilitinı» hưuuunh htin (Ego, Ich, Self l ujLiq) (nt'́u, nuluufjuir 1995p. 11): Zuulu-

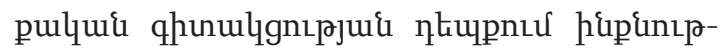

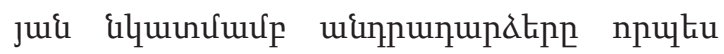

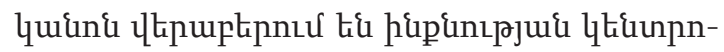

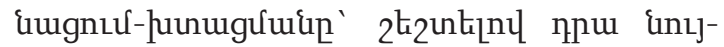

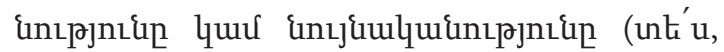

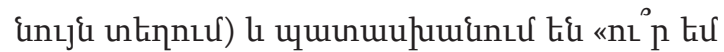

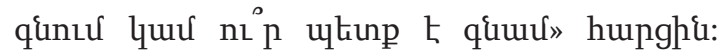

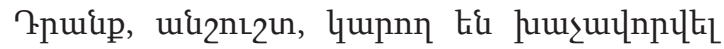
ptí pnsq hungtiph, ptí dulqupnulqutph (qh-

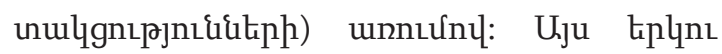

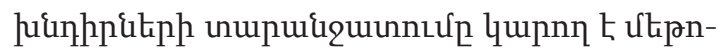

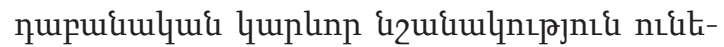

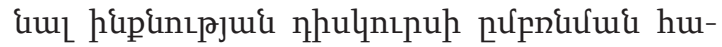
uup: Ophiuul' Onnpphuir ujnophaul «nupuiquin punupulquinıpjuis» ไun npulnpnıu t. uhis Upupnıppe Onıpphujh hưưp

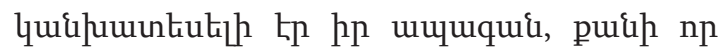

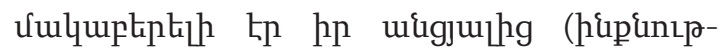
jnılihg): Upurpntpphg uluud' Onıpphui

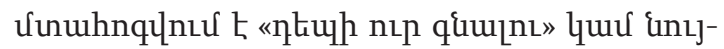
quiluinıpjui hungtinnц:

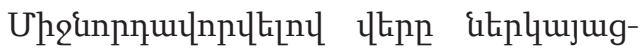

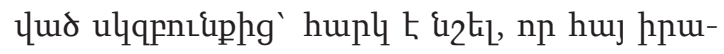
quínıpjuis utis hưuru ujn tiplng nh-

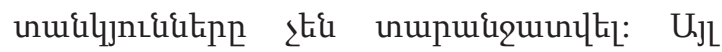

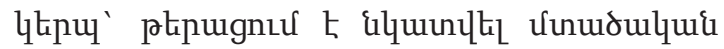
uje tplinujph qhunulgưue hungnuर, np

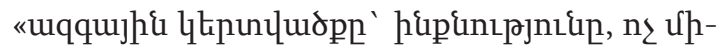

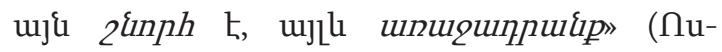
quijuif 1995u. 8):
Apultu paupui \&lipluujuglur Stipjuid-

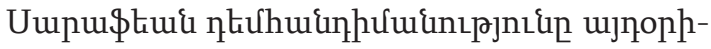
aul tiplnıpjui jnıpophaul upunuhuj-

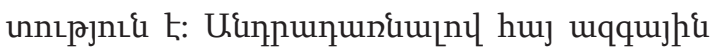

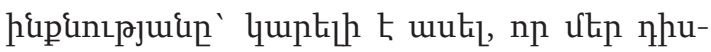

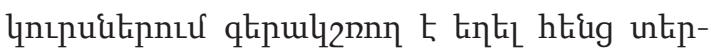

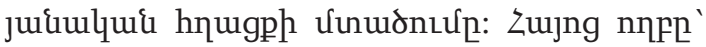
Unlutiu funptiaugnı w2humunnıpuid utig wn-

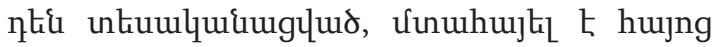

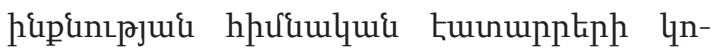

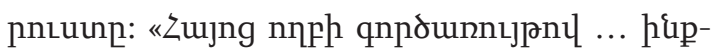

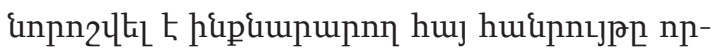

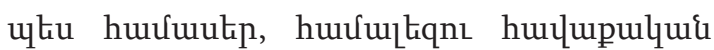

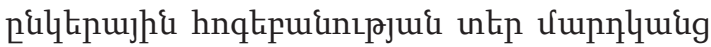
Uhnıpjnı\{» (Uunqunjui 2002. 61): Uuцuðh

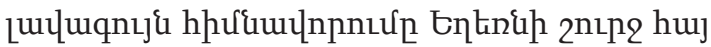

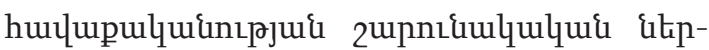

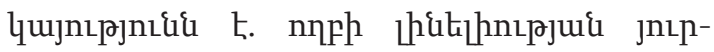
ophiuul «ququpdaultiun», npny pluuunltig

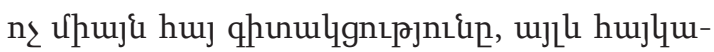
quinıpjnı\&\& wnhuuwpuly:

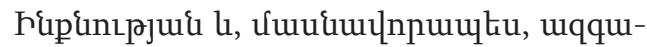

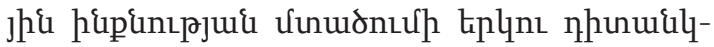

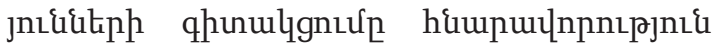

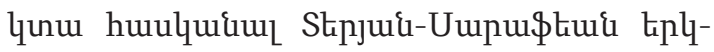

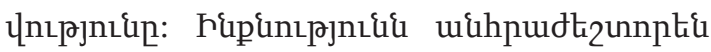
unlqu t kplyns huppnıpjnı\&qupnnư durư-

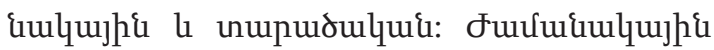

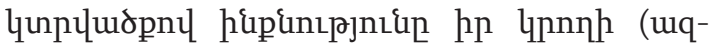

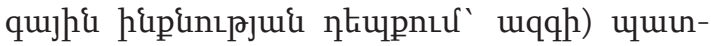

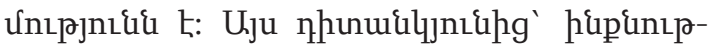

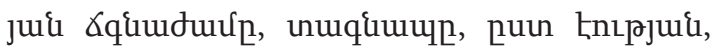

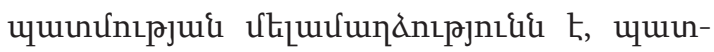

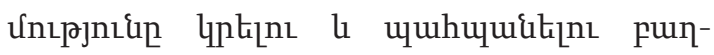

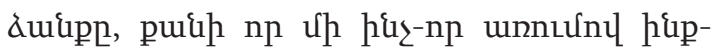

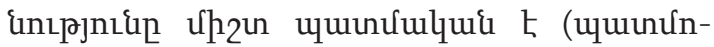

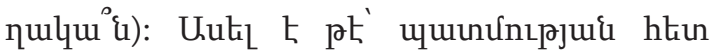

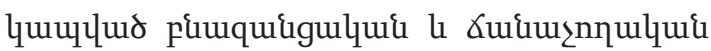

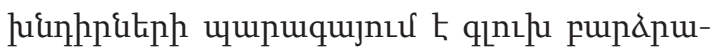
ginıu hipanıpjui hungn: Utihuluid ưun- 


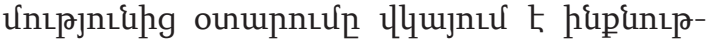

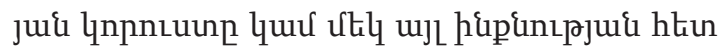

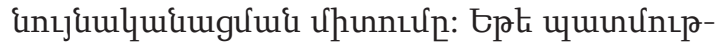

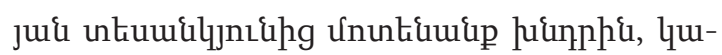

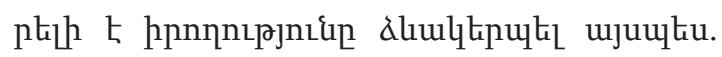

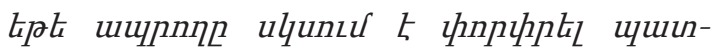

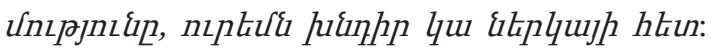

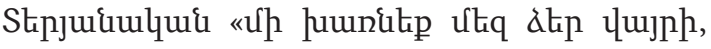
ungh gtinkpha»-n, puu knıpjuid, umunưu-

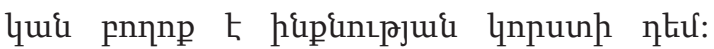

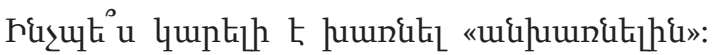
El hisut:

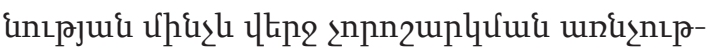
jưuf ounuphis utinunpuip unuewnptip:

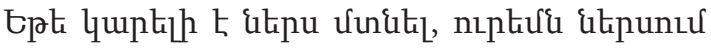
utin qu: Rzuun tipte ptiq 24nptęn pugum-

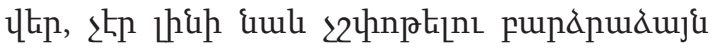

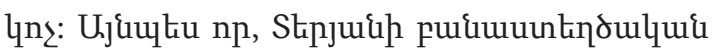

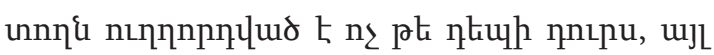

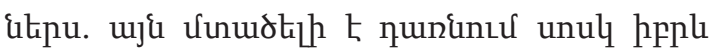

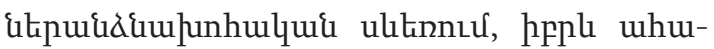

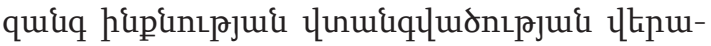
ptrpuul:

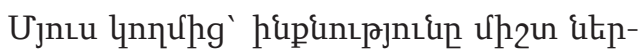

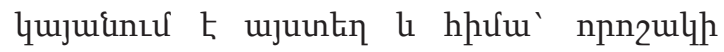

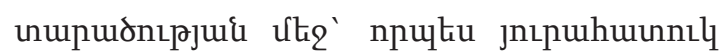

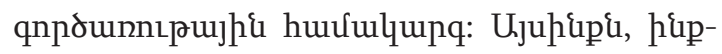

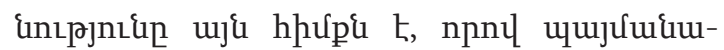

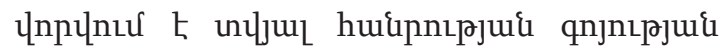

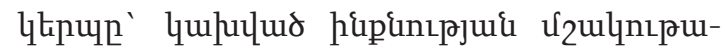

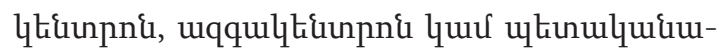
likunpni hilinng: Zujptiupp htiag hip\&nıpjui unqujugntud t: hul puip np

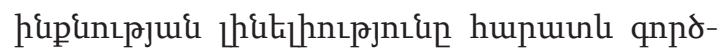

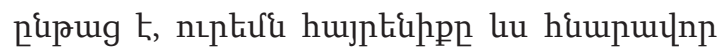

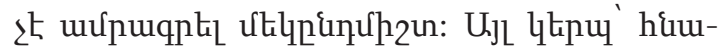

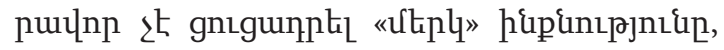

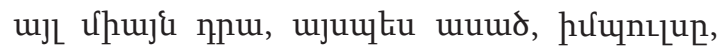

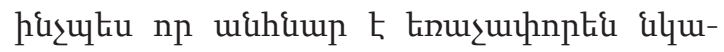

puqptil hujptippe (uju unphl untinhs $t$

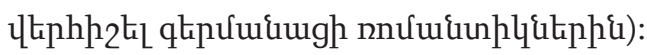

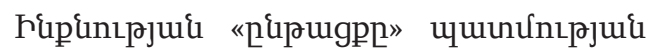

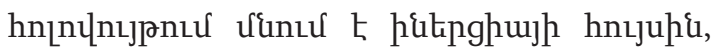

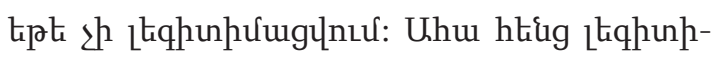

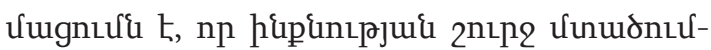
fitpp uhunh «uluquih» nL unuigp uupph:

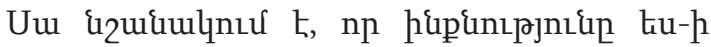

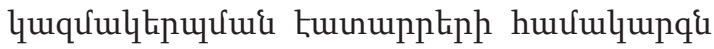

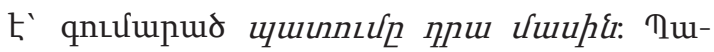

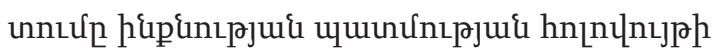

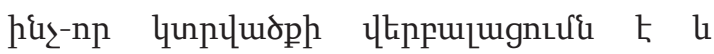

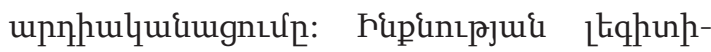

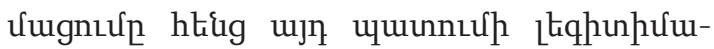

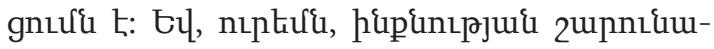

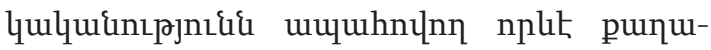

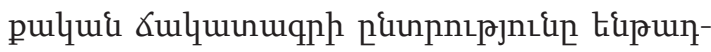

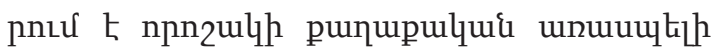

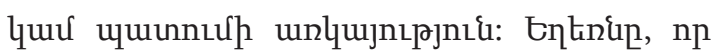

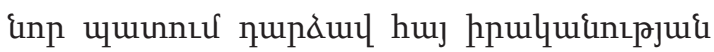
Utį li hujh hipanıpjuí unupp nupåuul,

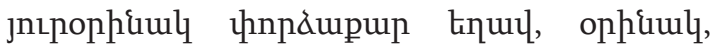

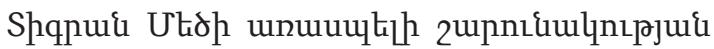

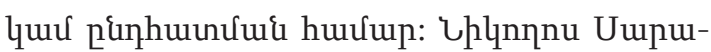

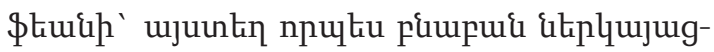

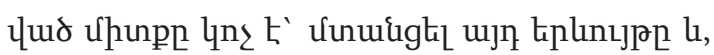
oqunuqnpttinıl huj hipanıpjuis unupu-

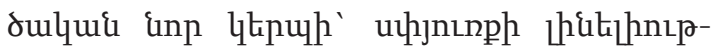

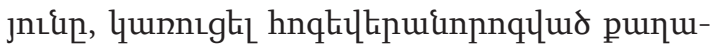

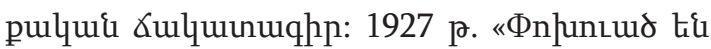
nupknp» tuuknıu Uupuqtiuin qpnsu Ł.

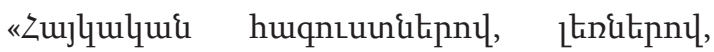

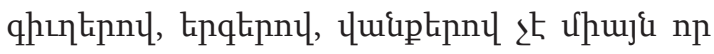

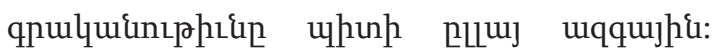

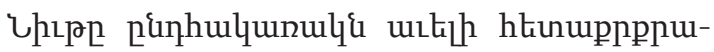

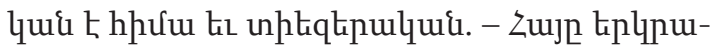

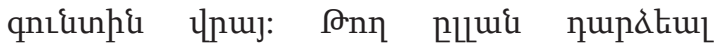

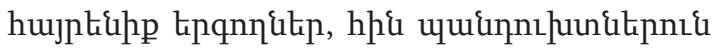

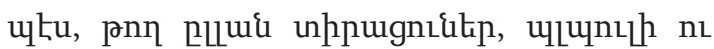

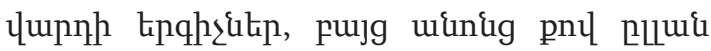




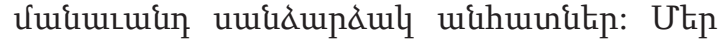

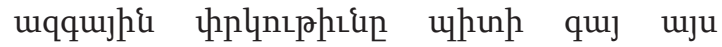

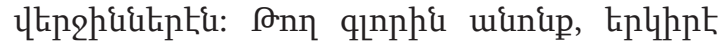

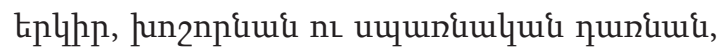

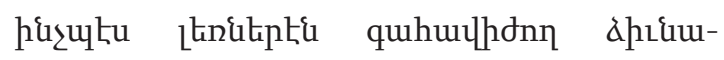

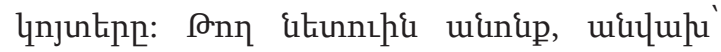

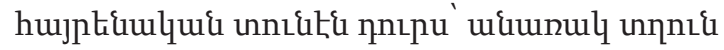

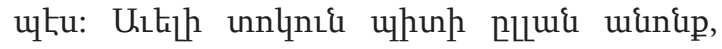

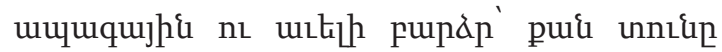

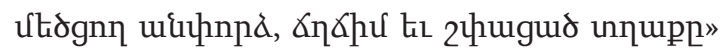
(Uupuqtiui 1994. 14):

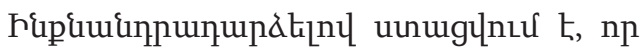

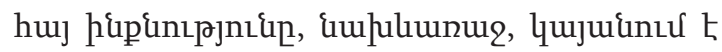
ưuunnufitennud, npnig hhưpe hujuqh-

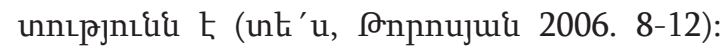

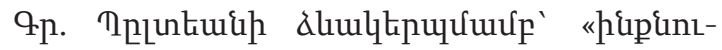

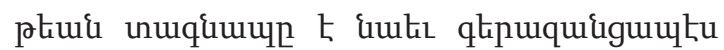
qpulquis unuqfaum» (Tplutiuis 2002. 93): qpuluinıрjnı\&p le hujuqhunnıpjnı\&p unhuuupuly uhong tis (ptiplue tquilh)'

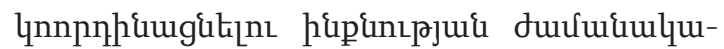

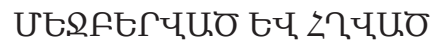

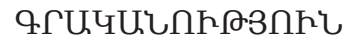

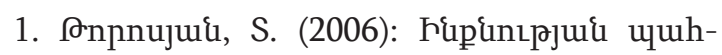

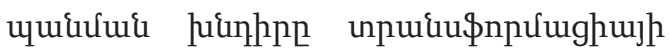
qnponipurgnıu // Lpupkp huuupulququid qhunnıpjnıqutiph, Ep., 2006, No1, te 523:

2. Uupqupjux, U. (2002): Uqqujhi Unum-

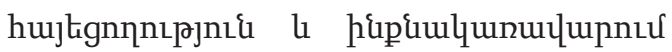

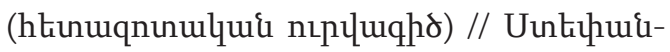

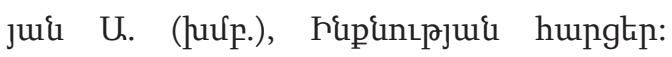
Epluui:

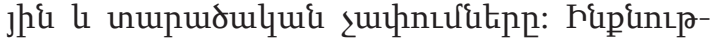

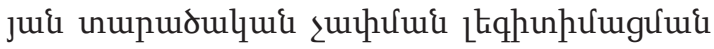

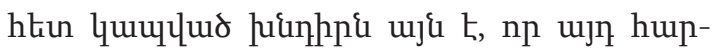
pnıpjuis utis humumuplnn pninp lnnutipn huintiu tis quihu uqqh, hujptinpp que

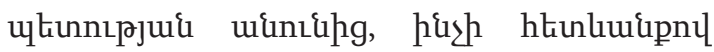

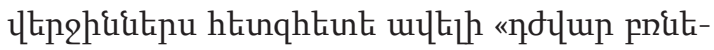

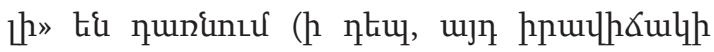

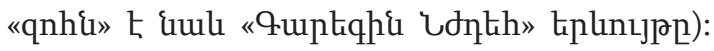

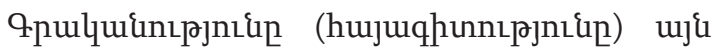

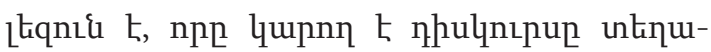

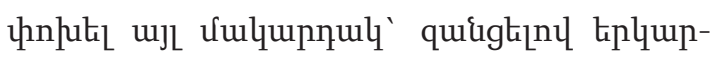

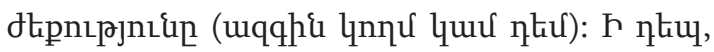

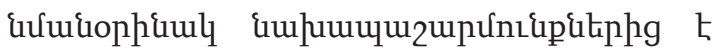
zujuuunuip ungl quiqquer fntumunum-

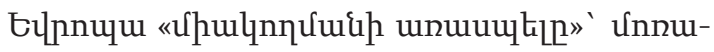

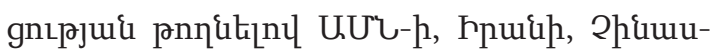

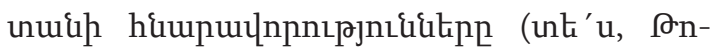
pnujuif 2006. 13): Uhizntin nppuid hiztin ne

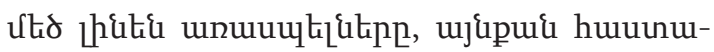

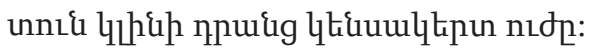

3. nuluufjuid, U. (1995w): 'umhuupuq // hip\{nıpjnı\{: Ep., «Yuưup»:

4. nuluufuuid, U. (1995p): Uqqujhi hipinıp-

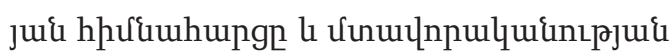

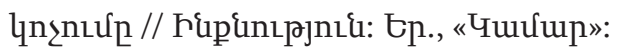

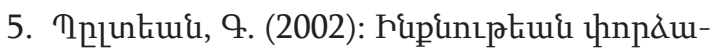

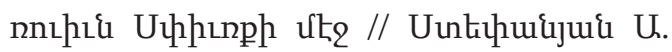
(hưf.), hipintpjui hungtip: Epluwi:

6. Uupuqtiuf, ᄂ. (1994): Stumpuiditen, uunnhl tu tu: Ephtua:

7. Huntington, S. (1993). The Clash of Civilizations? // Foreign Affairs; Summer 1993; 72, 3. 


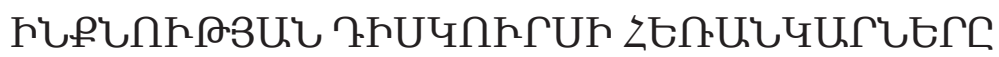

\section{(Utipnnupuqualqui nhunuplynuर्ukin)}

\section{UUФกФПНU}

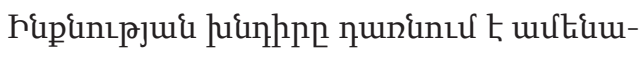

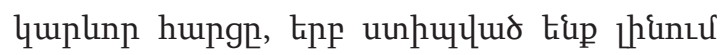

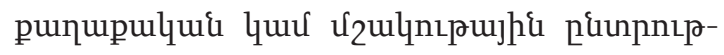

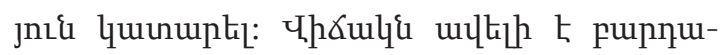
ansu, tep $\mathrm{h}$ hujun tis quihu punu-

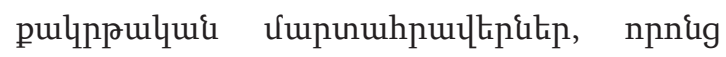

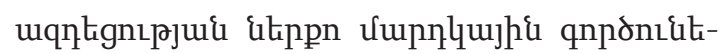

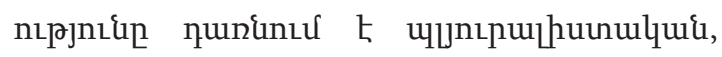

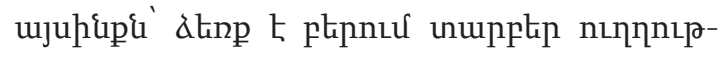

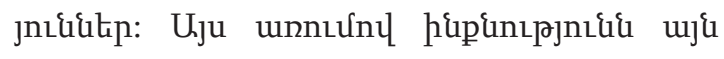

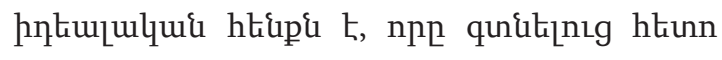

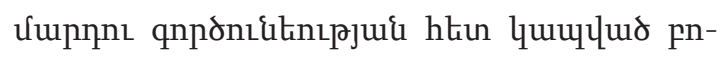

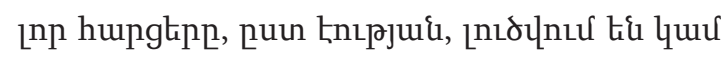

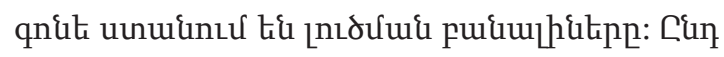

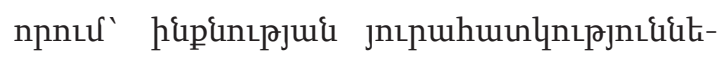

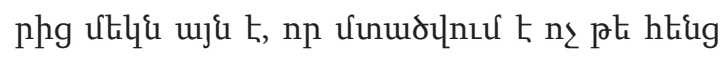

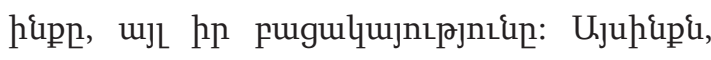

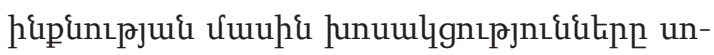

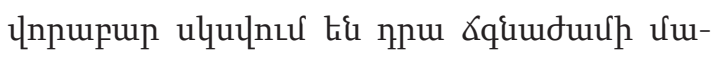
uhi hunuwlgnıpjnı\&\&iphg:

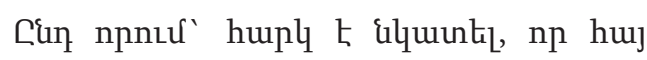
hpulquinıpjuis utis hưue hipinıpjui

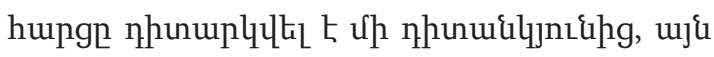

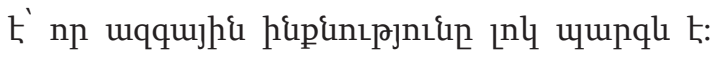

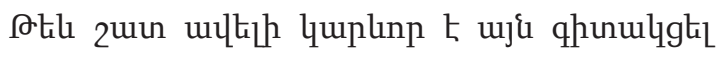
hppl unuqunpuip: El uhuje ujp

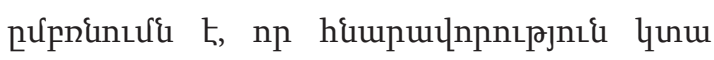
unuig zunduli!:

Zuiqnıgujh\& huulugnıpjnı\&itip. hip-

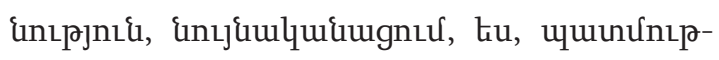
jnı\&, nhulnıpu:

Davit MOSINYAN

\section{THE PERSPECTIVES OF THE IDENTITY DISCOURSE}

\section{(methodological observations)}

\begin{abstract}
The identity issue is becoming the most important problem when we are forced by political or cultural choice. The situation is further complicated when there are civilizational challenges under the influence of what human activities become pluralistic, i.e. get different dimensions. From this point of view, the
\end{abstract}

ABSTRACT

identity is an ideal base, after finding of what all other aspects of human activity, in fact, are solved or at least get the keys to the solution. Meanwhile, one of the outstanding features of the identity is that one does not meditate on identity, but rather on its absence. In other 
words, the conversations about identity usually start with the crisis of identity.

Moreover, it should be noticed that the issue of identity is often considered as if the national identity is nothing more than a gift. While, it is more important to realize it as a task. And only this consideration will give an opportunity to move forward.

Key concepts: identity, identification, I, history, discourse.

Давид МОСИНЯН

\section{ПЕРСПЕКТИВЫ ДИСКУРСА ИДЕНТИЧНОСТИ (методологические замечания)}

\section{РЕЗЮМЕ}

Проблема идентичности становится наиболее важным вопросом, когда мы вынуждены делать политической или культурной выбор. Ситуация осложняется, когда есть цивилизационные вызовы, под влиянием которого деятельность человека становится плюралистической, т.е. приобретает разные измерения. В связи с этим, идентичность является той идеальной базой, выявляя чего все вопросы, касающиеся человеческой деятельности, на самом деле, решаются или, по крайней мере, получают ключи к решению. Между тем, одна из особенностей идентичности состоит в том, что человек вдумыва- ется не в идентичности, но, скорее, в его отсутствие. Иными словами, разговоры о личности, как правило, начинаются о кризисе идентичности.

При этом, следует отметить, что вопрос о личности в наших кругах часто обсуждается с одной точки зрения, а именно, что национальная идентичность не более, чем награда. Хотя более важно осознать идентичность как задачу. И только это понимание даст возможность двигаться вперед.

Ключевые понятия: идентичность, идентификация, я, история, дискурс. 